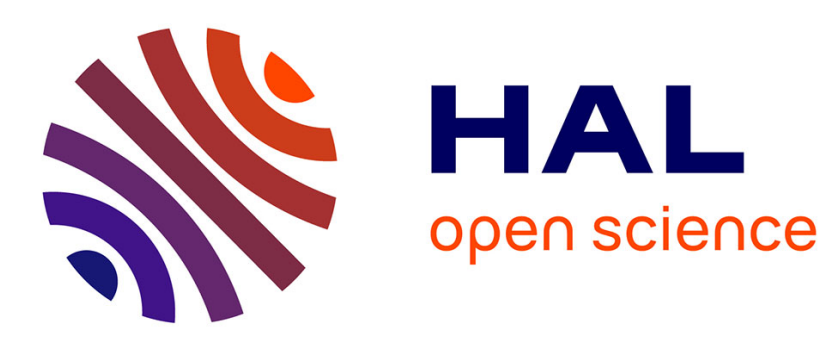

\title{
Performance evaluation of fuzzy-based fusion rules for tracking applications
}

\author{
A. Tchamova, J. Dezert
}

\section{To cite this version:}

A. Tchamova, J. Dezert. Performance evaluation of fuzzy-based fusion rules for tracking applications. International Journal of Reasoning-based Intelligent Systems, 2014, pp.1-9. 10.1504/IJRIS.2014.066251 . hal-01096450

\section{HAL Id: hal-01096450 https://hal.science/hal-01096450}

Submitted on 17 Dec 2014

HAL is a multi-disciplinary open access archive for the deposit and dissemination of scientific research documents, whether they are published or not. The documents may come from teaching and research institutions in France or abroad, or from public or private research centers.
L'archive ouverte pluridisciplinaire HAL, est destinée au dépôt et à la diffusion de documents scientifiques de niveau recherche, publiés ou non, émanant des établissements d'enseignement et de recherche français ou étrangers, des laboratoires publics ou privés. 


\title{
Performance evaluation of fuzzy-based fusion rules for tracking applications
}

\author{
A. Tchamova* \\ Institute of Information and Communication Technology, \\ Bulgarian Academy of Science, Bulgaria \\ E-mail: tchamova@bas.bg \\ *Corresponding author
}

\section{J. Dezert}

The French Aerospace Lab, Chemin de la Hunière, F-91761 Palaiseau, France

E-mail: jean.dezert@onera.fr

\begin{abstract}
:
The objective of this paper is to present and to evaluate the performance of particular fusion rules based on fuzzy T-Conorm/T-Norm operators for two tracking applications: (1) Tracking object's type changes, supporting the process of objects' identification (e.g. fighter against cargo, friendly aircraft against hostile ones), which, consequently is essential for improving the quality of generalized data association for targets' tracking; (2) Alarms identification and prioritization in terms of degree of danger relating to a set of a priori defined, out of the ordinary dangerous directions. The aim is to present and demonstrate the ability of these rules to assure coherent and stable way for identification and to improve decision-making process in a temporal way. A comparison with performance of Dezert-Smarandache Theory based Proportional Conflict Redistribution rule no.5 and Dempster's rule is also provided.
\end{abstract}

Keywords: objects' type identification; alarm classification; data fusion; DSmT, TCN rules, PCR5 fusion rule, Dempster's rule.

Reference to this paper should be made as follows: Performance evaluation of fuzzybased fusion rules for tracking applications, A. Tchamova, J. Dezert

Biographical notes: Albena Tchamova is an Associate Professor at the Department of Mathematical Methods for Sensor Information Processing, Institute for Information and Communication Technologies, Bulgarian Academy of Sciences. She received M.Sc. degree in Microelectronics and Ph.D. degree in Radiolocation and Navigation from the Technical University of Sofia, Bulgaria, in 1978 and 1998 respectively. Her main research interests include Information Fusion, Dezert-Smarandache Theory (DSmT) of Plausible and Paradoxical Reasoning, Decision Making under Uncertainty, Conflicts and Paradoxes.

Jean Dezert is Senior Research Scientist in the Information Modeling and Processing Dept. at the French Aerospace Lab (ONERA), Palaiseau, France. He got his Ph.D. from Univ. Paris XI, Orsay, France in 1990 in Automatic Control and Signal Processing. During 1991-1992, he visited the Dept. of ESE, UConn, Storrs, USA as ESA Postdoctoral Research Fellow. During 1992-1993 he was teaching assistant in EE Dept, University of Orléans, France. His current research interests include information fusion (IF) and plausible reasoning with applications to classification, decision-making support for defense and security, robotics, and risk assessment. Jean Dezert has published three books and more than a hundred of papers in conferences and journals on tracking and information fusion. He has given seminars and workshops in IF and tracking in USA, Canada, Europe, Australia and China, and he is reviewer for several international journals. He is Associate Editor of Journal of Advances in Information Fusion. 


\section{INTRODUCTION}

Surveillance system is intended to provide monitoring of observed area, in order to recognize and to track suspicious targets, as well to provide a smart operational control, based on the intelligent analysis and interpretation of alarms coming from a variety of sensors installed in the area. The general purpose is to prevent, instead of investigating dangerous situations, by providing relevant and accurate timely processed information for decisions, facilitating an appropriate evidence-based action. Targets' type estimates can be used during the tracking process stages for improving generalized data (attributes and kinematics) association and for the quality evaluation of complicated situations characterized with closely spaced or/and crossing targets (Bar Shalom, 1990; Blackman and Popoli, 1999; Bogler, 1987). It supports the process of identification, e.g. friendly aircraft against hostile ones, fighter against cargo. In such a case, although the attribute of each target is invariant over time, at the attribute-tracking level the type of the target committed to the (unresolved) track varies with time and must be tracked properly in order to discriminate how many different targets are hidden in the same unresolved track. Alarms classification and prioritization (Khosla and Dillon, 1997; Vale and Machado, 1993; Lin et al, 2004; DeSouza et al, 2004; McArthur et al, 1996; Foong et al, 2009) is very challenging task, because in case of multiple suspicious signals (relating to a set of a priori defined, out of the ordinary dangerous directions), generated from a number of sensors in the observed area, it requires the most dangerous among them to be correctly recognized, in order to decide properly where the video camera should be oriented. There are cases, when some of the alarms generated could be incorrectly interpreted as false, increasing the chance to be ignored, in case when they are really significant and dangerous. That way the critical delay of the proper response could cause significant damages. In both cases above, the uncertainty and conflicts encountered in objects' and signals' data, could weaken or even mistake the respective surveillance system decision. That is why a strategy for an intelligent, scan by scan processing and synergistic combination of data generated is needed in order to provide the surveillance system with a meaningful output providing a better understanding of the problem under consideration.

In this paper we focus our attention on the ability of alternative class fusion rules, the so called T-ConormNorm (TCN) fusion rules (Tchamova, Dezert, Smarandache, 2006), based on particular fuzzy operators, focusing on the t-norm based conjunctive rule as an analog of the ordinary conjunctive rule of combination and on tconorm based disjunctive rule as an analog of the ordinary disjunctive rule of combination. These rules are defined within Dezert-Smarandache Theory (DSmT) of plausible and paradoxical reasoning and are based on the Proportional Conflict Redistribution rule no.5 (PCR5).

Copyright (c) 200x Inderscience Enterprises Ltd.
The goal is to improve the process of data fusion and to successfully finalize the decision-making procedures in both described above surveillance cases.

The work is based on preliminary research in (Dezert and Smarandache, 2004-2009; Tchamova and Dezert, 2013). The basics of Proportional Conflict Redistribution rule no.5 (PCR5), defined within DSmT are recalled in Section II. Basics of PCR5 based TCN fusion rules are outlined in section III. The main properties, as well as different important types of t-norm and t-conorm operators are presented. In section IV the decision making approach used for both estimation procedures are described. The performance of TCN rules related to the problem of target type tracking is analyzed in section V. Section VI presents the problem of alarms' classification and examine the ability of TCN fusion rule to solve it. Performance, obtained in the most probable case, when sound signals emitted from the localized sources start asynchronously is also considered and discussed. A comparison with performances of Dezert-Smarandache Theory based Proportional Conflict Redistribution rule no.5 and Dempster's rule is also provided. Concluding remarks are given in section VII.

\section{BASICS OF PCR5 FUSION RULE}

The general principle of Proportional Conflict Redistribution rules is to: 1) calculate the conjunctive consensus between the sources of evidences; 2) calculate the total or partial conflicting masses; 3) redistribute the conflicting mass (total or partial) proportionally on non-empty sets involved in the model according to all integrity constraints. The idea behind the Proportional Conflict Redistribution rule no. 5 defined within DSmT (Dezert and Smarandache, 2004-2009) (Vol. 2) is to transfer conflicting masses (total or partial) proportionally to non-empty sets involved in the model according to all integrity constraints. Under Shafer's model assumption of the frame $\Theta$, PCR5 combination rule for only two sources of information characterized by their basic belief assignments (bba's) $m_{1}($.$) and m_{2}($. is defined as: $m_{P C R 5}(\emptyset)=0$ and $\forall X \in 2^{\Theta} \backslash\{\emptyset\}$

$$
\begin{aligned}
& \sum_{\substack{m_{P C R 5} \\
X_{2} \in 2^{\Theta} \backslash\{X\} \\
X_{2} \cap X=\emptyset}}\left[\frac{m_{1}(X)^{2} m_{2}\left(X_{2}\right)}{m_{1}(X)+m_{2}\left(X_{2}\right)}+\frac{m_{2}(X)^{2} m_{1}\left(X_{2}\right)}{m_{2}(X)+m_{1}\left(X_{2}\right)}\right]
\end{aligned}
$$

All sets involved in the formula (1) are in canonical form. The quantity $m_{12}(X)$ corresponds to the conjunctive consensus, i.e:

$$
m_{12}(X)=\sum_{\substack{X_{1}, X_{2} \in 2^{\Theta} \\ X_{1} \cap X_{2}=X}} m_{1}\left(X_{1}\right) m_{2}\left(X_{2}\right) .
$$

All denominators are different from zero. If a denominator is zero, that fraction is discarded. No matter how big or small is the conflicting mass, PCR5 mathematically 
does a better redistribution of the conflicting mass than Dempster-Shafer's rule since PCR5 goes backwards on the tracks of the conjunctive rule and redistributes the partial conflicting masses only to the sets involved in the conflict and proportionally to their masses put in the conflict, considering the conjunctive normal form of the partial conflict. PCR5 is quasi-associative and also preserves the neutral impact of the vacuous belief assignment.

Remark: Along with PCR5 fusion rule, the Proportional Conflict Redistribution no. 6 (PCR6) rule was defined within DSmT (Dezert and Smarandache, 2004-2009), Vol.2. PCR5 and PCR6 fusion rules coincide for the combination of two basic belief assignments, but they differ in general as soon as three or more sources have to be combined altogether, because of different principles of the proportional conflict transfer. In (Smarandache, Dezert, 2013) it is shown and proved the strong relationship between PCR6 rule and the averaging fusion rule which is commonly used to estimate the probabilities in the classical frequentist interpretation of probabilities. Such a probability estimate cannot be obtained using DempsterShafer (DS), nor PCR5 fusion rules. In this paper, we are only concerned with the fusion of two bba's, and so we use PCR5 fusion rule in our analysis which, in this case, coincides with PCR6.

\section{BASICS OF TCN FUSION RULE}

In this work, the combination rules for information fusion take their source from t-norm and t-conorm operators in fuzzy logics, where $A N D$ logic operator corresponds in information fusion to the conjunctive rule and $O R$ logic operator corresponds to the disjunctive rule. One interprets the fusion/association between the sources of information as vague relations, characterized with the following two characteristics:

- The way of association between the possible propositions is based on union and intersection operations.

- The degree of association between the propositions is obtained by using a t-norms (for conjunction) or tconorms (for disjunction) operators applied over the probability masses of corresponding focal elements. While the logic operators deal with degrees of truth and false, the fusion rules deal with degrees of belief of hypotheses.

TCN rules are defined within DSmT based PCR5 fusion rule. Under Shafer's model assumption of the frame $\Theta$, TCN fusion rules for only two sources of information are defined as: $\tilde{m}_{T C N}(\emptyset)=0$, and $\forall X \in 2^{\Theta} \backslash\{\emptyset\}$

$$
\begin{array}{r}
\tilde{m}_{T C N}(X)=\tilde{m}_{12}(X)+ \\
\sum_{\substack{X_{2} \in 2^{\Theta} \backslash\{X\} \\
X_{2} \cap X=\emptyset}}\left[\frac{m_{1}(X) \cdot \text { Tnorm }\left\{m_{1}(X), m_{2}\left(X_{2}\right)\right\}}{\text { Tconorm }\left\{m_{1}(X), m_{2}\left(X_{2}\right)\right\}}+\right. \\
\left.\frac{m_{2}(X) . \text { Tnorm }\left\{m_{2}(X), m_{1}\left(X_{2}\right)\right\}}{\text { Tconorm }\left\{m_{2}(X), m_{1}\left(X_{2}\right)\right\}}\right]
\end{array}
$$

The quantity $\tilde{m}_{12}(X)$ corresponds to the conjunctive consensus, obtained by:

$$
\tilde{m}_{12}(X)=\sum_{\substack{X_{1}, X_{2} \in 2^{\Theta} \\ X_{1} \cap X_{2}=X}} \text { Tnorm }\left\{m_{1}\left(X_{1}\right), m_{2}\left(X_{2}\right)\right\} .
$$

TCN fusion rules require a normalization procedure :

$$
\tilde{m}_{T C N}(X)=\frac{\tilde{m}_{T C N}(X)}{\sum_{\substack{X \in 2^{\Theta} \\ X \neq \oslash}} \tilde{m}_{T C N}(X)}
$$

Depending on the choice of a t-norms and t-conorms one obtains different results for conjunction and disjunction. Our goal is to study the way how different t-norms and t-conorms functions used in TCN fusion rule affects over the target type estimation and alarms classification performance.

The attractive features of TCN rules could be defined as: very easy to implement, satisfying the impact of neutral Vacuous Belief Assignment; commutative, convergent to idempotence, reflects majority opinion, assures adequate data processing in case of partial and total conflict between the information granules. The general drawback of these rules is related to the lack of associativity, which is not a main issue in temporal data fusion.

\subsection{Main Properties and Types of T-Norm and T-Conorm Functions}

Triangular norms (t-norms) and triangular conorms (tconorms) are the most general families of binary functions that satisfy the requirements of the conjunction and disjunction operators, respectively. They are twoplace functions mapping the unit square into the unit interval, i.e. T-norm : $[0,1]^{2} \mapsto[0,1]$ and $T$-conorm $:[0,1]^{2} \mapsto[0,1]$. They are monotonic, commutative and associative. tnorms have to satisfy the following conditions:

- Associativity:

$\operatorname{Tnorm}(\operatorname{Tnorm}(x, y), z)=\operatorname{Tnorm}(x, \operatorname{Tnorm}(y, z))$.

- Commutativity:

$$
\operatorname{Tnorm}(x, y)=\operatorname{Tnorm}(y, x)
$$

- Monotonicity:

$$
\text { if }(x \leq a) \&(y \leq b) \text { then Tnorm }(x, y) \leq \operatorname{Tnorm}(a, b)
$$


- Boundary condition: the number 1 becomes the neutral element for t-norms:

$$
\operatorname{Tnorm}(0,0)=0 ; \quad \operatorname{Tnorm}(x, 1)=x
$$

There are many functions satisfying t-norm conditions, among which the most important are:

- Minimum operator, also called Gödel t-norm, as it is the standard semantics for conjunction in Gödel fuzzy logic. It occurs in most t-norm based fuzzy logics as the standard semantics for weak conjunction:

$$
m(X)=\min \left\{m_{1}\left(X_{i}\right), m_{2}\left(X_{j}\right)\right\}
$$

- Algebraic product operator, which is the standard semantics for strong conjunction in fuzzy logic:

$$
m(X)=m_{1}\left(X_{i}\right) \cdot m_{2}\left(X_{j}\right) .
$$

- Lukasiewicz operator:

$$
m(X)=\max \left\{\left[m_{1}\left(X_{i}\right)+m_{2}\left(X_{j}\right)-1\right], 0\right\}
$$

The dual notion of t-norm is called t-conorm. The conditions to satisfy are the same as for t-norms, but the neutral element, which is $0(T \operatorname{conorm}(x, 0)=x)$ instead of 1 , as in t-norms. The important dual t-conorms, corresponding to the above described t-norms are:

- Maximum Gödel operator - it is the smallest t-conorm and is used to represent the weak disjunction in all tnorm based fuzzy logics.

$$
m(X)=\max \left\{m_{1}\left(X_{i}\right), m_{2}\left(X_{j}\right)\right\} .
$$

- Probabilistic sum, which is the standard semantics for strong disjunction:

$$
m(X)=m_{1}\left(X_{i}\right)+m_{2}\left(X_{j}\right)-m_{1}\left(X_{i}\right) \cdot m_{2}\left(X_{j}\right) .
$$

- Bounded sum operator, the standard semantics for strong disjunction in Lukasiewicz fuzzy logic:

$$
m(X)=\min \left\{\left[m_{1}\left(X_{i}\right)+m_{2}\left(X_{j}\right)\right], 1\right\} .
$$

\section{Decision-making support}

In this work, we assume Shafer's model and the classical Pignistic Transformation (Dezert and Smarandache, 20042009 ) is used here to take a decision. This subjective probability measure (also called betting probability) is defined for $\forall A \in 2^{\Theta}$ by:

$$
\operatorname{BetP}(A)=\sum_{X \in D^{\Theta}} \frac{|X \cap A|}{|X|} \cdot \tilde{m}(X)
$$

where $|X|$ denotes the cardinality of $X$.
In this work, the estimation of targets' types as well as the level of alarms' danger at time $k$ are given by the most probable model, i.e. the one having the maximum of pignistic probability $\operatorname{Bet} P($.$) . However, \operatorname{Bet} P($.$) is not$ the best solution to approximate a non-Bayesian bba into a subjective probability measure because the value of its PIC (Probabilistic Informational Content) is not very high in general and that is why it is recommended to use the $D S m P($.$) transformation as explained in details in (Dezert$ and Smarandache, 2004-2009), Vol.3. DSmP(.) takes into account both the values of the belief masses and the cardinality of focal elements in a proportional redistribution process of masses committed to partial ignorances and thus $D S m P($.$) increases the PIC values and reduces the entropy$ of the subjective probability measure approximated from the bba. In this work, we didn't use $\operatorname{DSmP(.)}$ because it was a bit more complex to implement and mainly because the main problem for correct alarm identification doesn't come from the specific probabilistic transformation used, but mainly from the choice of the fusion rule applied. An inefficient fusion rule will provide bad results which can never be corrected by a method (even a very good one like $D S m P$ ) to approximate the bba into subjective probability measure for decision-making.

\section{TARGET TYPE TRACKING APPROACH}

The principle of our estimators is based on the sequential combination of the current basic belief assignment (drawn from classifier decision, i.e. our measurements) with the prior bba estimated up to current time from all past classifier declarations.

The problem can be simply stated as follows:

- Let $k=1,2, \ldots, k_{\max }$ be the time index and consider $M$ possible target types $T_{i} \in \Theta=\left\{\theta_{1}, \ldots, \theta_{M}\right\}$ in the environment; for example $\Theta=\{$ Fighter, Cargo $\}$ and $T_{1} \triangleq$ Fighter, $T_{2} \triangleq$ Cargo.

- at each instant $k$, a target of true type $T(k) \in \Theta$ (not necessarily the same target) is observed by an attribute-sensor (we assume a perfect target detection probability here).

- the attribute measurement of the sensor (for example noisy Radar Cross Section) is then processed through a classifier which provides a decision $T_{d}(k)$ on the type of the observed target at each instant $k$.

- The sensor is in general not totally reliable and it is characterized by a $M \times M$ confusion matrix:

$$
\mathbf{C}=\left[c_{i j}=P\left(T_{d}=T_{j} \mid \text { TrueTargetType }=T_{i}\right)\right]
$$

The goal is to estimate $T(k)$ from the sequence of declarations done by the unreliable classifier up to time $k$, i.e. how to build an estimator $\hat{T}(k)=$ $f\left(T_{d}(1), T_{d}(2), \ldots, T_{d}(k)\right)$ of $T(k)$. The principle of the 
estimator is based on the sequential combination of the current basic belief assignment (drawn from classifier decision, i.e. our measurements) with the prior bba estimated up to current time from all past classifier declarations.

The algorithm follows the next main steps:

1 Initialization step (i.e. $k=0$ ). Select the target type frame $\Theta=\left\{\theta_{1}, \ldots, \theta_{M}\right\}$ and set the prior bbas $m^{-}$.) $\left(\tilde{m}_{T C N}^{-}(\right.$.$) for TCN rule, m_{P C R 5}^{-}($.$) for PCR5 rule, and$ $m_{D S}^{-}($.$) for DS rule) as vacuous belief assignment, i.e$ $m^{-}\left(\theta_{1} \cup \ldots \cup \theta_{M}\right)=1$ since one has no information about the first target type that will be observed.

2 Generation of the current bba $m_{\text {obs }}($.$) from the cur-$ rent classifier declaration $T_{d}(k)$ based on attribute measurement. At this step, one takes $m_{\text {obs }}\left(T_{d}(k)\right)=$ $c_{T_{d}(k) T_{d}(k)}$ and all the unassigned mass $1-m_{o b s}\left(T_{d}(k)\right)$ is then committed to total ignorance $\theta_{1} \cup \ldots \cup \theta_{M}$.

3 Combination of current bba $m_{\text {obs }}($.$) with prior bba$ $m^{-}$(.) to get the estimation of the current bba $m($.$) .$ Symbolically we will write the generic fusion operator as $\oplus$, so that $m()=.\left[m_{o b s} \oplus m^{-}\right]()=.\left[m^{-} \oplus m_{o b s}\right]($.$) .$ The combination $\oplus$ is done according either TCN fusion rules $\left(m()=.m_{T C N}().\right)$, or PCR5 rule $(m()=$. $\left.m_{P C R 5}().\right)$, ot Demspter's rule $\left(m()=.m_{D S}().\right)$.

4 Estimation of True Target Type is obtained from $m($. by taking the singleton of $\Theta$, i.e. a Target Type, having the maximum of belief (or eventually the maximum Pignistic Probability).

5 set $m^{-}()=.m($.$) ; do k=k+1$ and go back to 2$)$.

\subsection{Simulations results}

In order to evaluate the performances of TCN fusion rules based estimators, a set of Monte-Carlo simulations on a very simple scenario for a $2 \mathrm{D}$ Target Type frame, i.e. $\Theta=\{(F)$ ighter, $(C)$ argo $\}$ is realized for classifier with a following confusion matrix:

$$
\mathbf{C}=\left[\begin{array}{ll}
0.9 & 0.1 \\
0.1 & 0.9
\end{array}\right]
$$

We assume there are two closely spaced targets: Cargo and Fighter. Due to circumstances, attribute measurements received are predominately from one or another and both targets generates actually one single (unresolved kinematics) track. To simulate such scenario, a Ground Truth sequence over 100 scans was generated. The sequence starts with the observation of a Cargo type and then the observation of the target type switches two times onto Fighter type during different time duration. At each time step $k$ the decision $T_{d}(k)$ is randomly generated according to the corresponding row of the confusion matrix of the classifier given the true target type (known in simulations). Then the algorithm from above is applied. The simulation consists of 10000 Monte-Carlo runs. The computed averaged performances (on the base of estimated belief masses obtained by the tracker) are shown on the figures 1 and 2 .

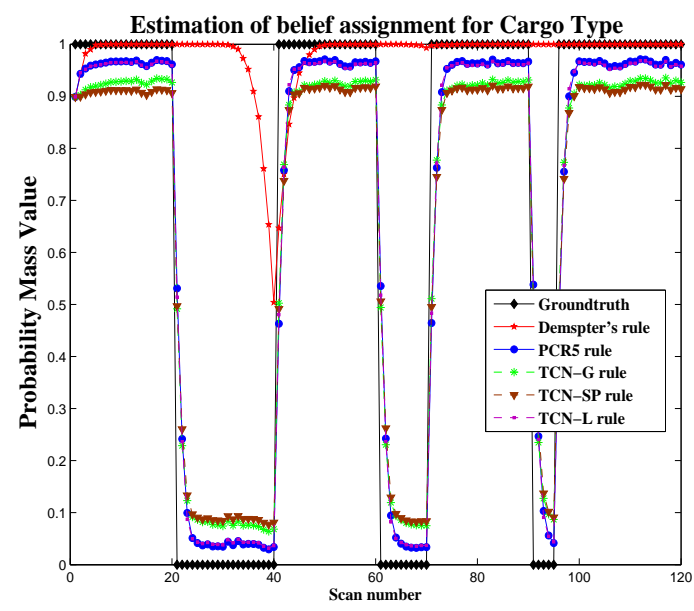

Figure 1: Estimation of belief assignment for Cargo type.

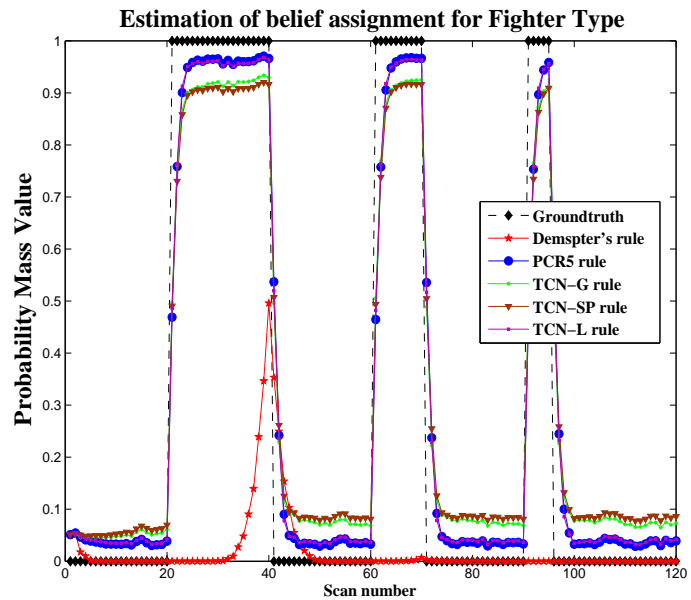

Figure 2: Estimation of belief assignment for Fighter type.

They are based on TCN fusion rule realized with different t-conorm and t-norm functions. On the same figures, for a comparison purposes, the respective performances of PCR5 and DS rule are presented. It is evident, that PCR5 fusion rule outperforms the results based on TCN rule, because PCR5 allows a very efficient Target Type Tracking, reducing drastically the latency delay for correct Target Type decision. TCN fusion rule shows a stable and adequate behavior, characterized with more smoothed process of re-estimating the belief masses in comparison to PCR5 $\mathrm{f}$. TCN fusion rule based on Lukasiewicz t-conorm and t-norm operators (denoted as TCN-L in the legend) reacts and adopts much better than TCN rule based on Gödel's t-conorm and t-norm operators (denoted as TCNG), followed by TCN rule, based on probabilistic Sum and Product for t-conorm and t-norm (denoted as TCN-SP). 


\section{ALARMS CLASSIFICATION APPROACH}

The approach assumes all the localized sound sources to be subjects of attention and investigation for being indication of dangerous situations. The specific input sounds' attributes, emitted by each source, are sensor's level processed and evaluated in timely manner for their contribution towards correct alarms' classification (in term of degree of danger). The applied algorithm considers the following steps:

- Defining the frame of expected hypotheses as follows: $\Theta=\left\{\theta_{1}=(E)\right.$ mergency, $\theta_{2}=(A)$ larm, $\theta_{3}=$ ( $W$ )arning $\}$. Here Shafer's model holds and we work on the power-set:

$2^{\Theta}=\{\emptyset, E, A, W, E \cup A, E \cup W, A \cup W, E \cup A \cup W\}$.

The hypothesis with a highest priority is Emergency, following by Alarm and then Warning.

- Defining an input rule base to map the sounds' attributes (so called observations) obtained from all localized sources into non-Bayesian basic belief assignments $m_{\text {obs }}($.$) .$

- At the very first time moment $k=0$ we start with a priori basic belief assignment (history) set to be a vacuous belief assignment $m_{\text {hist }}(E \cup A \cup W)=1$, since there is no information about the first detected degree of danger according to sound sources.

- Combination of currently received measurement's bba $m_{\text {obs }}($.$) (for each of located sound sources), based on$ the input interface mapping, with a history's bba, in order to obtain estimated bba relating to the current degree of danger $m()=.\left[m_{\text {hist }} \oplus m_{\text {obs }}\right]($.$) . TCN rule$ is applied in the process of temporal data fusion to update bba's associated with each sound emitter.

- Flag for an especially high degree of danger has to be taken, when during the a priori defined scanning period, the maximum Pignistic Probability (Dezert and Smarandache, 2004-2009) is associated with the hypothesis Emergency. In this work, we assume Shafer's model and we use the classical Pignistic Transformation (Dezert and Smarandache, 2004-2009), (Smets and Kennes, 1994) to take a decision about the mode of danger. It is defined for $\forall A \in 2^{\Theta}$ by:

$$
\operatorname{BetP}(A)=\sum_{X \in D^{\Theta}} \frac{|X \cap A|}{|X|} \cdot m(X)
$$

where $|X|$ denotes the cardinality of $X$.

\subsection{Simulation Scenario}

A set of three sensors located at different distances from the microphone array are installed in an observed area for protection purposes, together with a video camera (Behar et al., 2010).

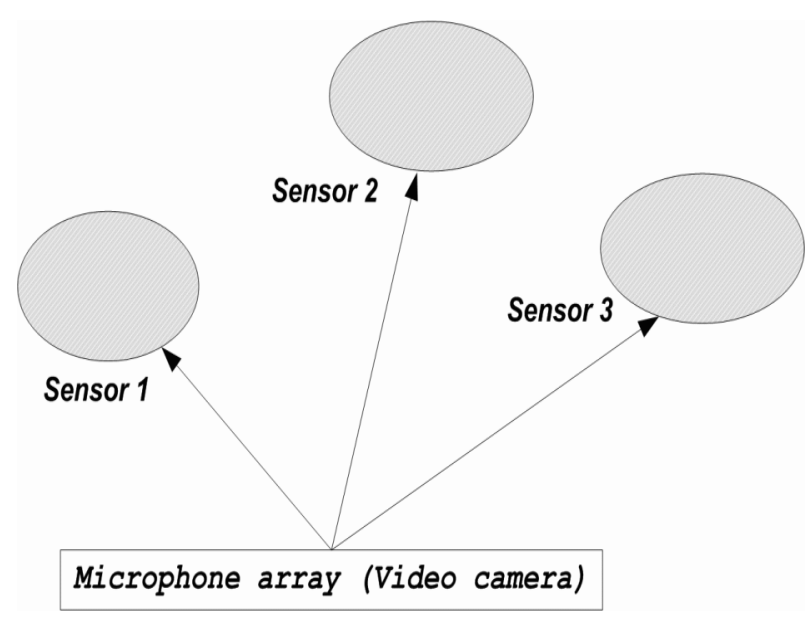

Figure 3: Simulation scenario.

They are assembled with alarm devices: Sensor 1 with Sonitron, Sensor 2 with E2S, and Sensor 3 with System Sensor. In case of alarm events (smoke, flame, intrusion, etc.) they emit powerful sound signals with various duration and frequency of intermittence (Table 1), depending on the nature of the event.

Table 1 Sound signal parameters.

\begin{tabular}{|c|c|c|}
\hline $\begin{array}{c}\text { Continuous } \\
(\text { Warning })\end{array}$ & $\begin{array}{c}\text { Intermittent-I } \\
(\text { Alarm })\end{array}$ & $\begin{array}{c}\text { Intermittent-II } \\
(\text { Emergency })\end{array}$ \\
\hline$f_{\text {int }}=0 \mathrm{~Hz}$ & $f_{\text {int }}=5 \mathrm{~Hz}$ & $f_{\text {int }}=1 \mathrm{~Hz}$ \\
$T_{\text {sig }}=10 \mathrm{~s}$ & $T_{\text {sig }}=30 \mathrm{~s}$ & $T_{\text {sig }}=60 \mathrm{~s}$ \\
\hline
\end{tabular}

The frequency of intermittencies $f_{\text {int }}$, associated with the localized sound sources is utilized in the specific input interface (the rule base) below.

Rule 1: if $f_{\text {int }} \rightarrow 1 H z$ then $m_{o b s}(E)=0.9$ and $m_{o b s}(E \cup$ A) $=0.1$.

Rule 2: if $f_{\text {int }} \rightarrow 5 \mathrm{~Hz}$ then $m_{\text {obs }}(A)=0.7, m_{\text {obs }}(A \cup E)=$ 0.2 and $m_{o b s}(A \cup W)=0.1$.

Rule 3: if $f_{\text {int }} \rightarrow 0 \mathrm{~Hz}$ then $m_{\text {obs }}(W)=0.6$ and $m_{\text {obs }}(W \cup$ $A \cup E)=0.4$.

Three main cases are estimated: the probabilities of modes, evaluated for Sensor 1 (associated with Emergency mode), Sensor 2 (associated with Alarm mode), and Sensor 3 (associated with Warming mode. The decisions should be governed at the video camera level, taken periodically, depending on: 1) specificities of the video camera (time needed to steer the video camera toward a localized direction); 2) time duration needed to analyze correctly and reliably the sequentially gathered information. We choose as a reasonable sampling period for camera decisions $T_{d e c}=20 s e c$, i.e. at every 10 th scan.

\subsection{TCN-L rule performance for danger level es- timation.}

TCN fusion rule based on Lukasiewicz t-conorm and tnorm operators is used here, because this rule shows the 

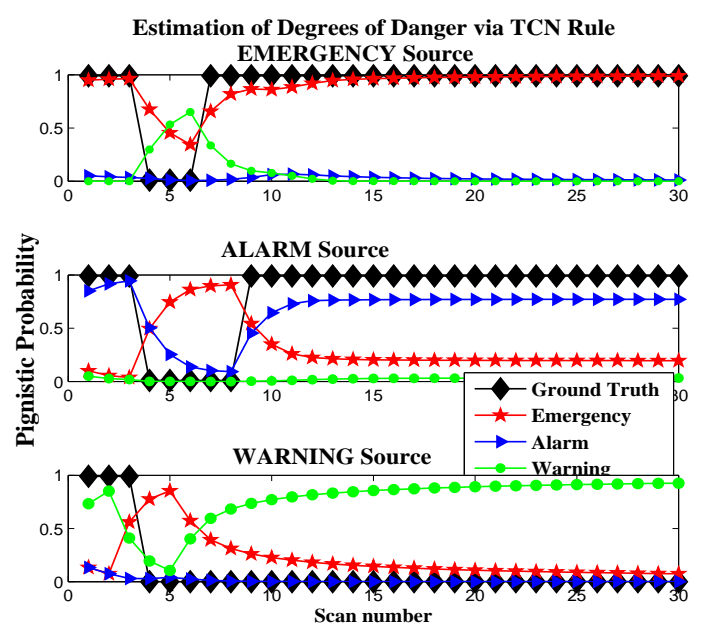

Figure 4: TCN rule Performance for danger level estimation.
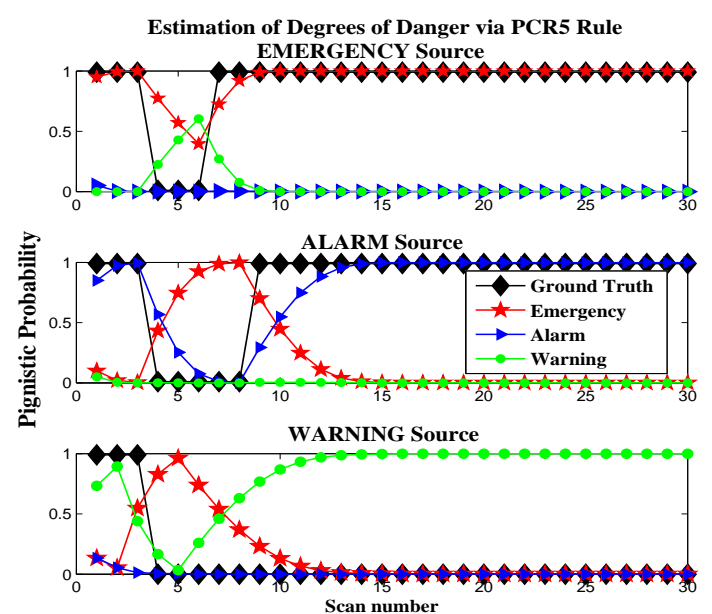

Figure 5: PCR5 rule Performance for danger level estimation.

best performance amongst all defined TCN rules in section III. Fig. 4 shows the values of Pignistic Probabilities of each mode $(E, A, W)$ associated with three sound emitters (1st source in $E$ mode, (subplot on the top), 2nd source in $A$ mode (subplot in the middle), and 3rd source in $W$ mode, (subplot in the bottom)) during the all 30 scans. Each source has been perturbed with noises in accordance with the simulated Ground Truth, associated with particular sound source. These probabilities are obtained for each source independently as a result of sequential data fusion of $m_{o b s}($.$) sequence using TCN combinational rule. For a$ completeness of study and for comparison purposes, the respective performances of PCR5 and DS rule are presented in fig. 5 and fig. 6 .

TCN rule shows a stable, quite proper and effective behavior, following the performance of PCR5 rule. A special feature of TCN rule performance are the smoothed estimates and more cautious decisions taken at the particular
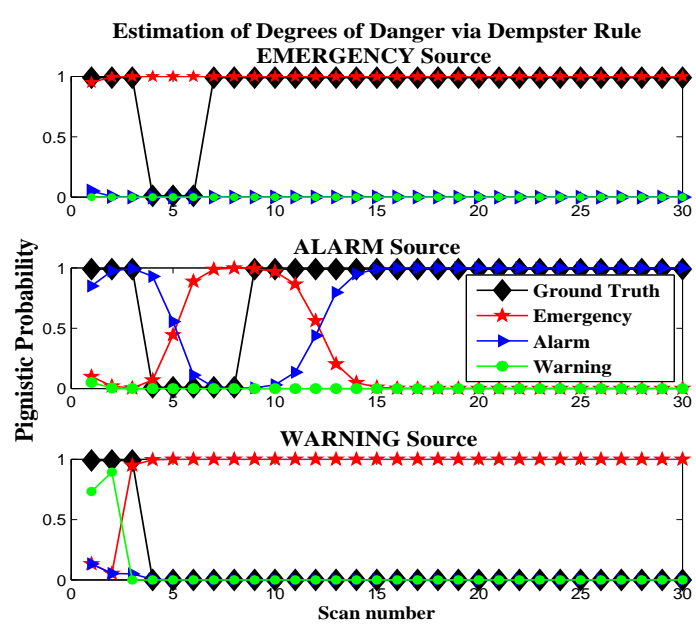

Figure 6: Dempster's rule Performance for danger level estimation.

decisive scans.

The results obtained show the strong ability of PCR5 rule to take care in a coherent and stable way for the evolution of all possible degrees of danger, related to all the localized sources. It is especially significant in case of sound sources data discrepancies and conflicts, when the highest priority mode Emergency occurs. PCR5 rule prevents to produce a mistaken decision, that way prevents to avoid the most dangerous case without immediate attention. A similar adequate behavior of performance is established in cases of lower danger priority. DS rule shows weakness in resolving the cases examined. In Emergency case, DS rule does not reflect at all new obtained informative observations supporting the Warning mode. This pathological behavior reflects the dictatorial power of DS rule realized by a given source (Dezert and Tchamova, 2014), which is fundamental in Dempster-Shafer reasoning (Shafer, 1976). In our particular case however, DS rule leads to a right final decision by coincidence, but this decision could not be accepted as coherent and reliable, because it is not built on a consistent logical ground. In cases of lower dangers priority (perturbed Warning and Alarm mode), DS rule could cause a false alarm and can deflect the attention from the existing real dangerous source by assigning a wrong steering direction to the surveillance camera.

\subsection{Performances obtained in asynchronous case.}

The most probable scenario concerns the case, when sound signals emitted from the localized sources start asynchronously. The corresponding figures 7,8 , and 9 show the values of Pignistic Probabilities of each mode (Emergency, Alarm, Warning) associated with the same three emitters. Source 1 starts emitting first, initializing scan 1 , source 2 becomes active at scan no. 5 and source 3 starts emitting at scan no. 10. The sampling period for decision-making for video camera steering remains $T_{\text {decision }}=10$ scans and the camera is switched on as soon as a sound is received 


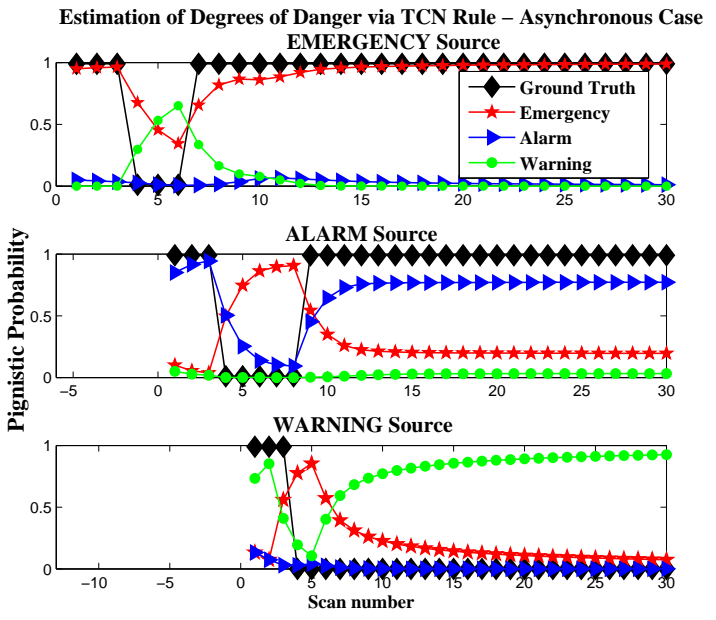

Figure 7: TCN rule Performance for danger level estimation.

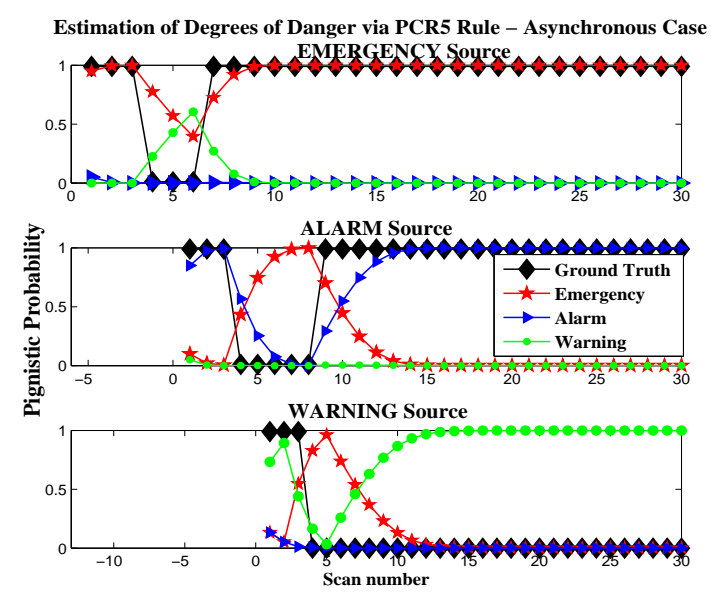

Figure 8: PCR5 rule Performance for danger level estimation.

(i.e. when source 1 starts to emit a signal). The decisions will be established based on the estimation of modes of source 1, source 2 and source 3 at scans 10, 20 and 30 .

In this asynchronous scenario and from the figures 7,8 and 9, one sees also the inadequate behavior of DS rule which fails to correctly estimate the true modes of the different sources and this will generate wrong prioritization of camera steering. In comparison, PCR5-based fusion approach allows also even in such case to get a better estimation of modes of sources and thus to obtain a more reliable alarm prioritization (as in the synchronous scenario).

\section{CONCLUSIONS}

In this paper, two tracking applications of a particular fuzzy fusion rule, based on fuzzy t-Conorm/t-Norm operators are presented: (1) Tracking Object's Type Changes, supporting the process of identification; (2) Alarms iden-

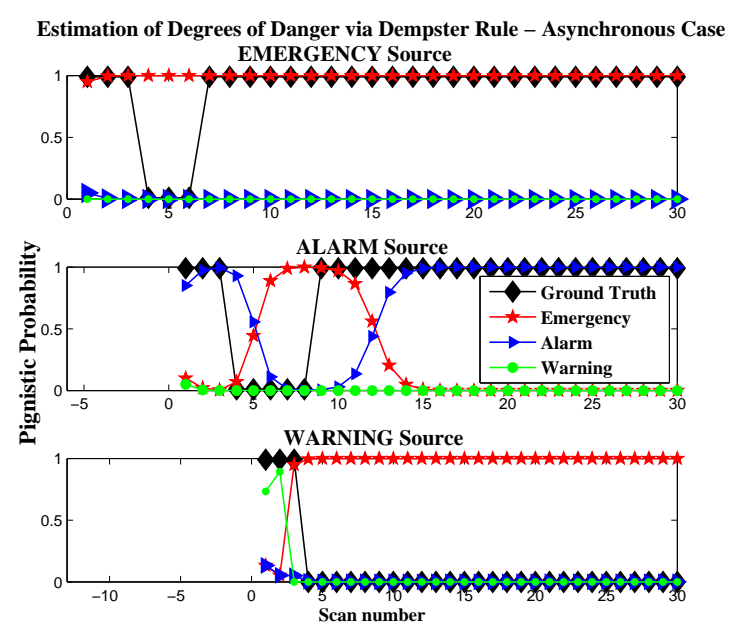

Figure 9: Dempster's rule Performance for danger level estimation.

tification and prioritization in terms of degree of danger relating to a set of a priori defined, out of the ordinary dangerous directions. The ability of TCN rule to assure coherent and stable way of identification and to improve decision-making process in temporal way are demonstrated. Different types of t-conorm and t-norms, available in fuzzy set/logic theory provide us with richness of possible choices to be used applying TCN fusion rule. The attractive features of TCN rule is it's easy implementation and adequate data processing in case of conflicts between the information granules.

\section{REFERENCES}

Bar-Shalom, Y. (2000) 'Multitarget-Multisensor Tracking: Advanced Applications', Artech House.

Blackman, S. and Popoli, R. (1999) 'Design and Analysis of Modern Tracking Systems', Artech House.

Bogler P.L. 'Shafer-Dempster reasoning with applications to multisensor target identification systems', IEEE Transactions on Systems, Man and Cybernetics, Vol. 17, No. 6, pp. 968-977.

Khosla, R. and Dillon, T. (1997) 'Learning knowledge and strategy of a neuro expert system architecture in alarm processing', IEEE Trans. Power Systems, Vol. 12, No. 4, pp. $1610-1618$.

Vale, Z. and Machado, A. (1993) 'An expert system with temporal reasoning for alarm processing in power system control centers', IEEE Trans. Power Systems, Vol. 8, No. 3, pp. 1307-1314.

Cignoli, Roberto L.O., D'Ottaviano, Itala M.L. and Mundici, D. (2000) 'Algebraic Foundations of Manyvalued Reasoning', Dordrecht: Kluwer, ISBN 0-7923$6009-5$. 
Lin, W. et al. (2004) 'Adaptive multiple fault detection and alarm processing for loop system with probabilistic network', IEEE Trans. Power Delivery, Vol. 19, No. 1, pp. 64-69.

Souza, J. et al. (2004) 'Alarm processing in electrical power systems through a neuro fuzzy approach', IEEE Trans. Power Delivery, Vol. 19, No. 2, pp. 537-544.

McArthur, S. et al. (1996) 'The application of model based reasoning within a decision support system for protection engineers', IEEE Trans. Power Delivery, Vol. 11, No. 4, pp. $1748-1754$.

Foong, O. et al. (2009) 'ALAP: Alarm Prioritization System For Oil Refinery', Proc. of the World Congress on Engineering and Computer Science, Vol. 2, San Francisco, CA, USA.

Smarandache, F. and Dezert, J. (Editors) (2004-2009) 'Advances and Applications of DSmT for Information Fusion', American Research Press, Rehoboth, Vol. 1-3,USA. San Francisco, CA, USA.

Tchamova, A., Dezert, J. (2013) 'Tracking applications with fuzzy-based fusion rules', IEEE International Symposium on Innovations in Intelligent Systems and Applications (INISTA), Albena, Bulgaria, pp. 5, June 19-21.

Tchamova, A., Dezert, J. and Smarandache, F. (2006) 'A New Class of Fusion Rules based on T-Conorm and TNorm Fuzzy Operators', Information and Security, An International Journal, Vol. 20, pp. 77-93.

Smarandache F., Dezert J., (2013) 'On the consistency of PCR6 with the averaging rule and its application to probability estimation', in Proc. of Fusion 2013 Int. Conf. on Information Fusion, Istanbul, Turkey, July 912, 2013.

Dezert, J. and Tchamova, A. (2014) 'On the validity of Dempster's fusion rule and its interpretation as a generalization of Bayesian fusion rule', International Journal of Intelligent Systems, Vol. 29, no.3, pp. 223-252.

Behar, V. et al. (2010) 'STAP Approach for DOA Estimation using Microphone Arrays', SPIE Proc. Signal Proc. Workshop, Vilnius, Vol. 7745.

Shafer, G. (1976) 'A Mathematical Theory of Evidence', Princeton University.

Smets, P. and Kennes, R. (1994) 'The transferable belief model', Artif. Intel, Vol. 66, No. 2, pp. 191-234.

Mendel, J. (1995) 'Fuzzy Logic Systems for Engineering: A Tutorial', Proc. of the IEEE, pp. 345-377. 